\title{
A Hybrid Framework for Coordinated Voltage Control of Power Systems
}

\author{
Mohammad Moradzadeh \\ SYSTeMS Research Group, Department of EESA \\ University of Ghent \\ Ghent, Belgium \\ Mohammad.Moradzadeh@ugent.be
}

\author{
René Boel \\ SYSTeMS Research Group, Department of EESA \\ University of Ghent \\ Ghent, Belgium \\ Rene.Boel@ugent.be
}

\begin{abstract}
Modeling and simulation of increasingly complex power systems is becoming more important for design, implementation and validation of power system on-line management. Current special-purpose tools are generally weak in the sense that they are mostly block-oriented and thus demand a huge amount of manual rewriting to get the equations into explicit form. Moreover, modification and examination of their encapsulated component models is very time-consuming and often practically impossible.

This paper describes an efficient hybrid framework for modeling and simulation of power systems in the interest of coordinated voltage control and stability analysis using Modelica as a generalpurpose object-oriented language. The proposed hybrid framework has been tested on a 12-bus power system. Simulation results show that the interaction between continuous dynamics of the power system and hybrid automata representing the discrete logical controllers and also nonlinear behavior of load dynamics can easily be studied in the proposed framework. On the other hand, the high speed of the simulator allows any coordinated control strategy to be effectively verified as countermeasure to arrest voltage collapse.
\end{abstract}

Keywords-modeling; simulation; hybrid automata; voltage control; LTC; OXL; Modelica; Dymola

\section{INTRODUCTION}

Traditional special-purpose tools e.g. PSCAD/EMTDC as well as general-purpose block-oriented tools e.g. Matlab/Simulink, for modeling and simulation of power systems are computationally very efficient and reasonably userfriendly, but their closed architecture makes them very timeconsuming and often practically impossible to examine or modify component models [1]. Component models should be as close as possible to the corresponding physical subsystems that build up the overall system, but due to causal modeling of these tools, the block-diagram structure cannot always reflect the actual topology of the physical system in the sense that some components can not be visible as an individual block and have to be combined into the model of other components and thus the proper understanding of the interaction between components becomes very difficult [2].

Causal modeling is a fundamental limitation of blockoriented tools at which the blocks have a unidirectional data flow from inputs to outputs. This is the reason why some components cannot be dealt with directly and if it is attempted to simulate the basic equations directly there will be a loop which only contains algebraic equations and that is a wellknown drawback of Matlab/Simulink which is not always able to handle the algebraic loops [3].

In order to be able to overcome the above-mentioned drawback, general-purpose object-oriented tools have been presented which are based on acausal modeling. Particularly, because of nonlinear hybrid behavior of power systems, the desired tool should also support the hybrid modeling. Hybrid behavior of power systems often involves an intrinsic strong coupling between continuous dynamics and discrete events, particularly during voltage collapse phenomena when many discrete devices (either controllers or thresholds) switch on and off. According to the literature, many voltage collapse incidents have been caused by uncoordinated interactions of local controllers following a major disturbance. In order to devise a truly coordinated voltage control combining wide-area communication with on-line optimization to get the optimal coordination of different control moves and automatic adaption to changing operating points due to the nonlinearity of power system, the desired simulator should also be able to capture these interactions.

This paper is organized as follows. Section II provides a brief discussion of useful features of Modelica for modeling and simulation of power systems. The model of components used for coordinated voltage control will be presented in section III using the proposed hybrid framework. These models are used, in section IV, to capture the hybrid behavior of a 12bus power system model and to investigate different countermeasures against voltage collapse following a major disturbance. Conclusions are provided in section V.

\section{MODELICA AND DYMOLA}

Modelica is a free general-purpose object-oriented equation-based language and has been designed to allow tools to generate very efficient codes for modeling of complex physical system. The modeling effort and complexity is considerably reduced in Modelica since the model of components can be reused avoiding tedious and error-prone manual manipulations.

There exist several free as well as commercial tools based on the Modelica language e.g. OpenModelica from OSMC, 
MathModelica by MathCore, SimulationX by ITI, MapleSim by MapleSoft and Dymola by Dassault systems/Dynasim[4]. Dymola, Dynamic Modeling Laboratory, is a powerful commercial simulation environment with the ability of dealing with huge systems described by more than hundred thousands equations containing a symbolic translator for Modelica equations generating $\mathrm{C}$ code for simulation. Graph theory is used to identify the variables to be solved in each equation and to find the minimal set of equations. The generated $\mathrm{C}$ code can via its convenient interfaces be transformed into a Matlab/Simulink S-function C mex-file which can be simulated in Matlab/Simulink as an input/output block. Modelica has two very important features which make it very efficient for modeling of power systems. These features are discussed in detail below.

\section{A. Acausal modeling}

In order to allow reuse of component models, the equations should be stated in a neutral form without consideration of computational order, meaning that a model's terminals do not necessarily have to be assigned an input or output role $[1,3]$.

Causality is generally not assigned in power systems. Setting the causality of an element of the power system involves representing the model equations in an explicit inputoutput state-space form required by Matlab/Simulink [5]. Often several manual steps including differentiation are required to transform the equations into this form. The need for manual transformations implies that it is cumbersome to build physics based model libraries in the block-based tools. A general solution to this problem requires a paradigm shift. Acausal modeling tools relax this causality constraint and allow focusing on the individual components and on the way these components are connected to each other by the topology of the system $[6,7]$. Modelica effectively supports acausal modeling.

\section{B. Hybrid modeling}

The behavior of power systems is characterized by the complex interactions between continuous dynamics of the power system and many hybrid automata representing the discrete logical controllers, i.e. power systems exhibit complex hybrid behavior and therefore their model is conveniently expressed in the following mixed discrete-event continuous differential-algebraic equations form:

$$
\begin{aligned}
& 0=\mathrm{g}(\mathrm{x}(\mathrm{t}), \mathrm{y}(\mathrm{t}), \mathrm{z}(\mathrm{t})) \\
& \dot{\mathrm{x}}(\mathrm{t})=\mathrm{f}(\mathrm{x}(\mathrm{t}), \mathrm{y}(\mathrm{t}), \mathrm{z}(\mathrm{t})) \\
& \mathrm{z}\left(\mathrm{t}_{\mathrm{k}}^{+}\right)=\mathrm{h}\left(\mathrm{x}\left(\mathrm{t}_{\mathrm{k}}{ }^{-}\right), \mathrm{y}\left(\mathrm{t}_{\mathrm{k}}{ }^{-}\right), \mathrm{z}\left(\mathrm{t}_{\mathrm{k}}{ }^{-}\right)\right), \mathrm{z}(\mathrm{t})=\mathrm{z}\left(\mathrm{t}_{\mathrm{k}}{ }^{+}\right) ; \mathrm{t}_{\mathrm{k}} \leq \mathrm{t}<\mathrm{t}_{\mathrm{k}+1}
\end{aligned}
$$

The dynamic continuous state vector $\mathrm{x}$ relates to synchronous generator, Automatic Voltage Regulator (AVR), Over eXcitation Limiter (OXL) and load dynamics. On the other hand, the algebraic state variables y relates to network voltages and currents via load flow equations with fast dynamics, and the discrete-event state variables $z\left(t_{k}\right)$ typically arise from discrete control logic such as thresholds reached by OXLs and logical controllers such as Load Tap Changing transformer (LTC), switched Capacitor Bank (CB) and disturbances. Modelica provides ordinary differential equations (ODEs) and differential-algebraic equations (DAEs) to mathematically describe the continuous time components model. It also supports several formalisms e.g. hybrid automata for modeling the evolution of the times when events occur.

\section{MODELING OF BASIC COMPONENTS OF POWER SYSTEM FOR COORDINATED VOLTAGE CONTROL ANALYSIS}

For the purpose of coordinated voltage control analysis, in the time scale of 0.1 second to several minutes, a power system will be advantageously considered as hybrid dynamical system and its basic components are modeled using Modelica. Continuous dynamics are expressed by DAEs and discrete events are modeled as hybrid automata. All component models are transparent and can easily be modified or extended. Notice that we aim at modeling of power system components in the hybrid framework taking discrete-events into account, while a basic Modelica library ObjectStab for power system stability studies has been proposed in [10].

\section{A. $\infty-b u s$}

By definition, an infinite bus refers to an infinitely strong rigid network with voltage and frequency unchanged under any load condition. Here it will be modeled as an ideal voltage source.

\section{B. Transmisson line}

Transmission lines can easily be modeled as $\pi$ - equivalent circuits but here for simplicity they will be modeled as series impedances. They will be considered more inductive in transmission systems but more resistive in distribution systems.

\section{C. $L T C$}

LTCs are slowly acting, discrete devices changing the transformer ratio by one step at a time if the voltage error remains outside a deadband around a reference voltage $v_{\text {ref }}$ longer than a specified time delay. The LTC thus controls the voltage of the connected bus. The LTC control system is modeled as a hybrid automaton shown in figure1.

The system remains in the state idle as long as the voltage deviation $\Delta \mathrm{v}$ is less than the chosen deadband. When the limit is exceeded, a transition to the state count occurs initializing a timer. This timer is kept running until either it reaches the detection delay time $\mathrm{T}_{\mathrm{d}}$, causing a transition to the state action, or until the voltage deviation becomes less than the deadband, causing a transition back to the state idle. When entering the state action, tap changer operation starts and after the mechanical delay time $T_{m}$, the tap position change is completed and the control system then returns to state idle [11].

Here LTC for simplicity will be modeled using an ideal transformer with variable $1: \mathrm{n}$ tap ratio in series with a pure leakage reactance $\mathrm{X}$. 


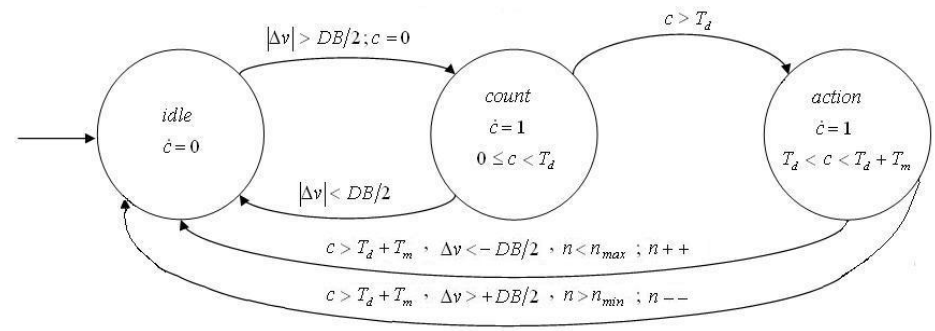

Figure 1. Hybrid automaton model for LTC

\section{Synchronous generator equipped with $A V R$ and $O X L$}

Synchronous generators are the primary source of active power and one of the main sources/sinks of reactive power in electrical power systems; therefore, they are to a great extent responsible for maintaining a good voltage profile across a power system. A general block-diagram representation of a synchronous generator equipped with first-order AVR and integral type OXL is shown in figure 2 [8].

The AVR controls the field current $\mathrm{I}_{\mathrm{fd}}$ to keep the terminal voltage of the synchronous generator close to the desired setpoint $\mathrm{V}_{0}$. The OXL protects the field winding from overheating due to excessive current by keeping $\mathrm{I}_{\mathrm{fd}}$ as close as possible to Ifd $_{\text {lim }}$ which is slightly larger than the permanent admissible field current. OXL activation has a direct effect on voltage support provided by the generator and it has to be included in the model of AVR for voltage instability studies.

Subtransient time constants which are a fraction of a second are negligible compared to the typical time interval of interest in voltage control and stability scenarios and a $3^{\text {rd }}$ order generator model accounting for the field winding only can effectively be used. Magnetic saturation is neglected for simplicity which is probably the most questionable simplification [8]. Assuming that the frequency and mechanical power are held constant, no governor will be considered.

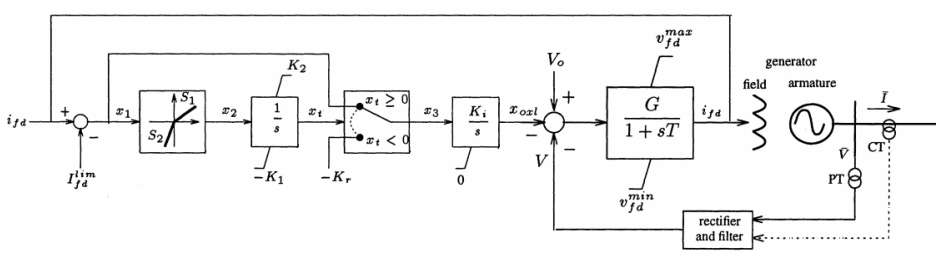

Figure 2. Block-diagram model of synchronous generator with overall AVR and OXL

The AVR is represented by the simple first-order transfer function with anti-windup limits on the field voltage. $G$ resp. T is the steady-state open-loop gain of the AVR resp. its related time constant. The translation of the OXL block-diagram implementation with inverse-time characteristic and integral action, shown in figure 2 , into a hybrid automaton model is shown in figure 3 . The hybrid automaton describing the OXL operation is decomposed into two smaller synchronously executing machines $S_{a}$ and $S_{b}$ which respectively implement inverse-time delay and limit enforcement by integral action. If $\mathrm{I}_{\mathrm{fd}}$ exceeds $\mathrm{Ifd}_{\mathrm{lim}}$, the OXL intermediate state variable $\mathrm{x}_{\mathrm{t}}$ starts increasing and as soon as it becomes positive, the error integration initializes and produces an $X_{\text {oxl }}$ signal that is subtracted from the AVR inputs causing $\mathrm{I}_{\mathrm{fd}}$ to decrease.
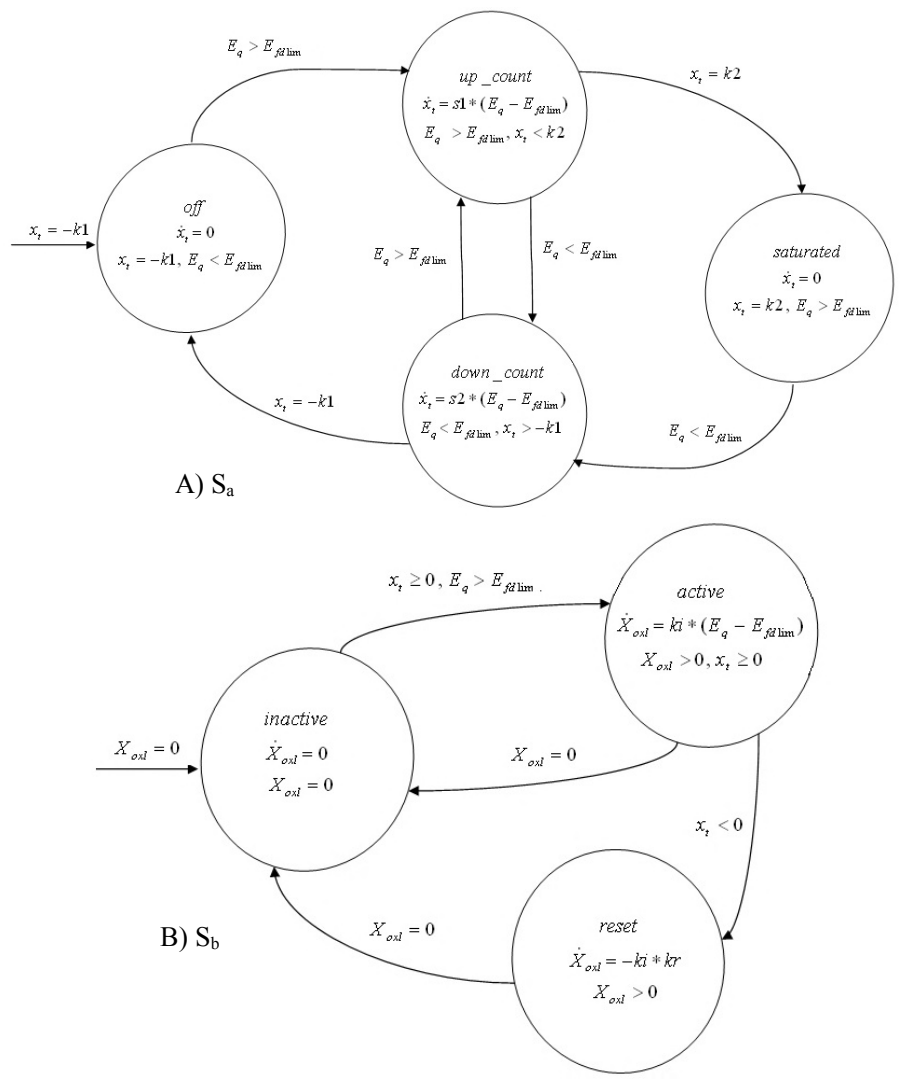

Figure 3. Hybrid automaton model for OXL A) inverse-time delay. B) limit enforcement by integral action

By way of summary, the continuous part of the synchronous generator model considered above as part of equation (1) can be written down in explicit matrix form as below.

$f(x(t), y(t), z(t))=\left[\begin{array}{c}\Delta \omega \\ \frac{1}{2 H}\left(P_{m}-P_{e}-D \Delta \omega\right) \\ -E_{q}^{\prime}+v_{f d}-\left(X_{d}-X_{d}^{\prime}\right) i_{d} \\ T_{d}^{\prime} \\ k_{i}\left(E_{q}^{\prime}+\left(X_{d}-X_{d}^{\prime}\right) i_{d}-E_{q l i m}\right) \\ \frac{-v_{f d}+G\left(V_{0}-V-x_{\text {oxl }}\right)}{T}\end{array}\right]$

where $\mathrm{x}=\left[\begin{array}{lll}\delta \Delta \omega \mathrm{E}_{\mathrm{q}}^{\prime} & \mathrm{x}_{\mathrm{oxl}} \mathrm{V}_{\mathrm{fd}}\end{array}\right]^{\mathrm{T}}$. The discrete-event part of the synchronous generator model was already represented in figure 3 . 


\section{E. Dynamic exponential recovery load}

The voltage dependence of loads is a key mechanism and driving force of voltage instability and for this reason voltage instability has also been called load instability. Load restoration is a process during which the dynamics of various load components (induction motors, thermostatic loads) and control mechanisms (including LTCs) tend to restore load power at least to a certain extent. Voltage instability results from the attempt of loads to draw more power than can be delivered by the transmission and generation system.

According to $[8,9]$ a so called additive generic selfrestoring load in which the load state variable is added to the transient characteristic will be modeled with an exponential type of voltage characteristic. The load dynamics of the additive generic model are given by the following differential equations.

$$
\begin{aligned}
& \dot{\mathrm{x}}_{\mathrm{p}}=-\frac{\mathrm{X}_{\mathrm{p}}}{\mathrm{T}_{\mathrm{p}}}+\mathrm{P}_{0}\left(\left(\frac{\mathrm{V}}{\mathrm{V}_{0}}\right)^{\alpha_{\mathrm{s}}}-\left(\frac{\mathrm{v}}{\mathrm{V}_{0}}\right)^{\alpha_{\mathrm{t}}}\right) \\
& \mathrm{P}=(1-\mathrm{k})\left(\frac{\mathrm{X}_{\mathrm{p}}}{\mathrm{T}_{\mathrm{p}}}+\mathrm{P}_{0}\left(\frac{\mathrm{v}}{\mathrm{v}_{0}}\right)^{\alpha_{\mathrm{t}}}\right) \\
& \dot{\mathrm{X}}_{\mathrm{q}}=-\frac{\mathrm{X}_{\mathrm{q}}}{\mathrm{T}_{\mathrm{q}}}+\mathrm{Q}_{0}\left(\left(\frac{\mathrm{V}}{\mathrm{V}_{0}}\right)^{\beta_{\mathrm{s}}}-\left(\frac{\mathrm{V}}{\mathrm{V}_{0}}\right)^{\beta_{\mathrm{t}}}\right) \\
& \mathrm{Q}=(1-\mathrm{k})\left(\frac{\mathrm{X}_{\mathrm{q}}}{\mathrm{T}_{\mathrm{q}}}+\mathrm{Q}_{0}\left(\frac{\mathrm{v}}{\mathrm{V}_{0}}\right)^{\beta_{\mathrm{t}}}\right)
\end{aligned}
$$

where,

P,Q: actual active resp. reactive power consumed by the load $\mathrm{P}_{0}, \mathrm{Q}_{0}$ : nominal load powers consumption

$\mathrm{v}_{0}$ : reference voltage

$\mathrm{T}_{\mathrm{p}}, \mathrm{T}_{\mathrm{q}}$ : active resp. reactive power recovery time constants

$\mathrm{x}_{\mathrm{p}}, \mathrm{x}_{\mathrm{q}}$ : continuous state variable of load dynamics

$\alpha_{s}, \beta_{\mathrm{s}}$ :steady-state active resp. reactive power voltage

dependency

$\alpha_{t}, \beta_{t}$ : transient active resp. reactive power voltage dependency

The scale factor of (1-k) on the load power has been introduced to model load shedding. No load shedding (full load) corresponds to $\mathrm{k}=0$, while complete load shedding is given by $\mathrm{k}=1$. In the case of any voltage drop on the load bus following a disturbance in power system, the load restoration process will initially start responding with its transient characteristics and the actual power consumed will drop instantaneously. Following this the load state variables $\mathrm{x}_{\mathrm{p}}$ and $\mathrm{x}_{\mathrm{q}}$ will start to increase causing both actual real and reactive power to recover to their steady-state characteristics. This process will end when either the steady-state characteristics are achieved or when the state variables reach their bounds.

\section{F. $\quad C B$}

Switched capacitor banks can locally support the voltage in connected bus. Each switching step of a CB corresponds to the injection of some reactive compensation which is quadratically dependent on the voltage, so it will provide less support at low voltages.

\section{SiMULATION RESUlTS}

The effectiveness of the proposed hybrid framework has been tested via some interesting experiments relating to coordinated voltage control on a realistic size 12-bus power system. This case study is taken from [11] and sets a control problem with around 20 control inputs, many measured disturbance inputs and up to 30 controlled outputs and many auxiliary outputs.

As shown in figure 4, the considered power system is composed of three almost identical areas connected together via three double tie lines as transmission system. The generators in Areas 2 and 3 are equipped with OXL modeled as in [8], while area 1 is fed by an $\infty$-bus. The distribution substation in each area is equipped with an LTC and a CB.

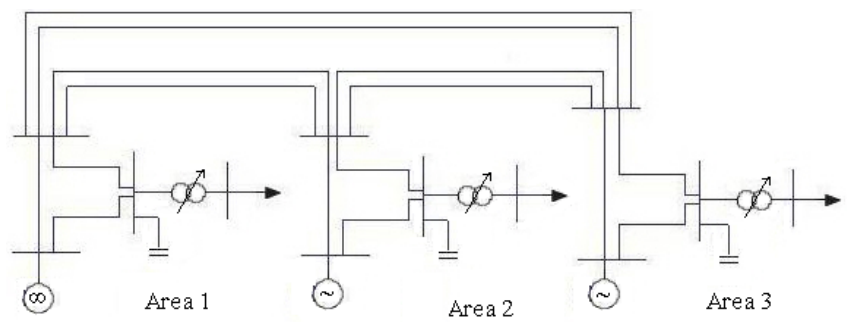

Figure 4. One-line diagram of a 12-bus power system

LTC and OXL constitute a primary control layer and our control objective is to design a secondary control layer to stabilize all bus voltages at values in the interval of $[0.9,1.1]$ p.u. by applying different countermeasures against voltage instability following the tripping of some transmission lines. The interaction between continuous dynamics of the power system and hybrid automata representing OXL and LTC and also the nonlinear behavior of load dynamics will be presented. Furthermore, it will be shown that in order to stabilize the bus voltages, the amount of loads to be shed can be minimized by a properly coordinated voltage control. Currently this strategy is determined by trail and error, but systematic search strategies, using the fast simulation tool, will be presented in a paper under preparation.

To have an idea about time scale values involved in this simulation, note that $\mathrm{T}_{\mathrm{d} 0}^{\prime}=8 \mathrm{~s}$ for synchronous generators, $\mathrm{T}=0.1 \mathrm{~s}$ for AVRs, $\mathrm{x}_{\mathrm{t}}=(20-100) \mathrm{s}$ for OXLs, $\mathrm{T}_{\mathrm{d}}+\mathrm{T}_{\mathrm{m}}=30 \mathrm{~s}$ for LTCs and $T_{p}=T_{q}=60 \mathrm{~s}$ for loads have been considered.

\section{A. No secondary control applied}

The load voltages, and the behavior of LTCs and OXLs are shown in figure 5 following the tripping of the double tie line between areas 1 and 3 at $\mathrm{t}=100 \mathrm{~s}$ where a standard uncoordinated primary control strategy is used.

Instability occurs and the solver fails to solve the non-linear equations of the system at $\mathrm{t}=652.3 \mathrm{~s}$ when simulation stops. Directly following the fault, load voltages in each area drop, slightly in area 1 compared to others, but soon after a shortterm equilibrium with all load voltages apparently settling down close to 1 p.u. is established. After this point the 
mechanism driving the system response is LTC together with load dynamics.

After the fault the generator field current in area 2 jumps to 2.13 p.u. which exceeds $\operatorname{Ifd}_{\text {lim }}=1.88$ p.u. for this generator. This initiates the inverse time characteristic of the OXL and eventually the OXL is activated at $\mathrm{t}=140.7 \mathrm{~s}$ meaning that the voltage support provided by this generator is withdrawn. This results in a further reduction of the load voltage causing the LTC to increase the tap position until the maximum tap limit is reached. Note that the integral type OXL forces the field current to Ifd $\mathrm{Iim}_{\mathrm{lim}}$ and subsequent tap changes result in a transient field current rise, which is quickly sensed and corrected by the OXL.
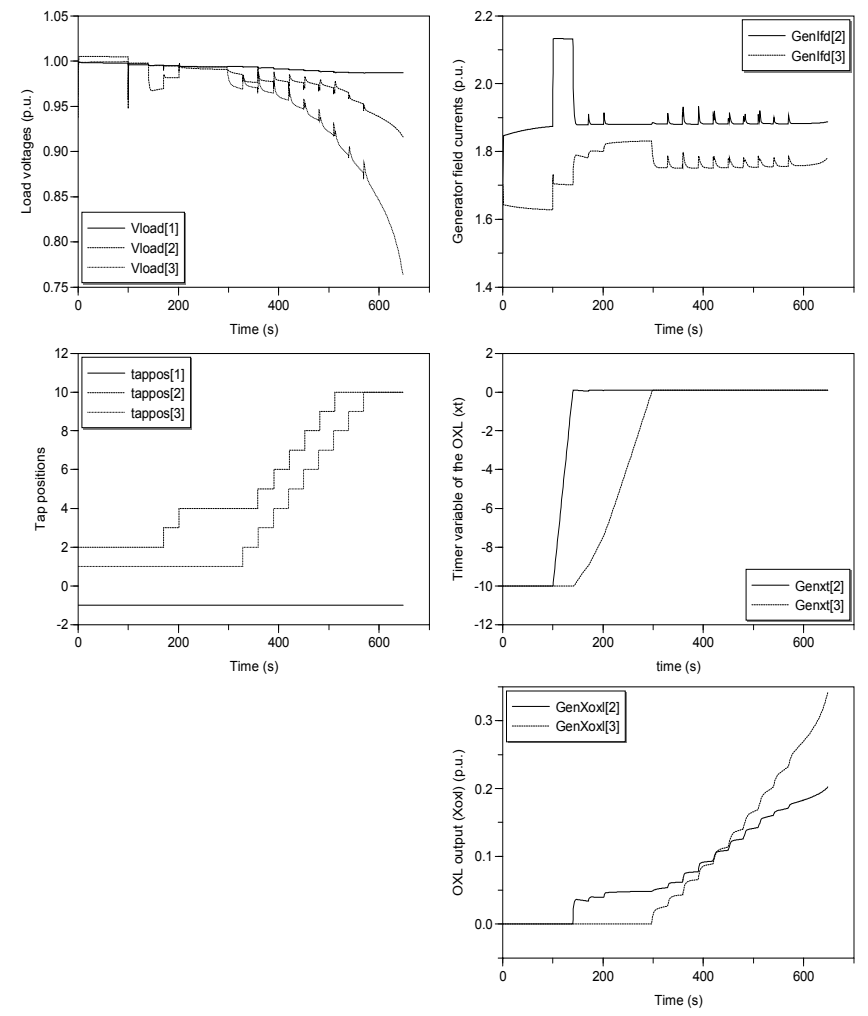

Figure 5. Response to the fault without secondary controller

\section{B. LTC setpoint reduction}

As the former experiment showed the LTC tap movements in area 2 and 3 aggravated further the load voltages profile and finally the load voltages collapsed dropping below 0.9 p.u. So, if the LTC tap movements can be somehow blocked or at least be slowed down, it seems that the voltage collapse possibly could be avoided or at least be delayed. Here, the load restoration process will be disabled by the reduction of the LTC setpoint voltage from 1 p.u. to 0.95 p.u. at $\mathrm{t}=150 \mathrm{~s}$ in both area 2 and 3. As shown in figure 6, this results in two downward tap movements for LTCs in both areas which relieves generator in area 3 of saturation and its field current is kept slightly below the limit $\operatorname{Ifd}_{\text {lim }}=1.75$ p.u. meaning that the related OXL will be inactivated in the long-term and as a result all load voltages are stabilized above 0.95 p.u.
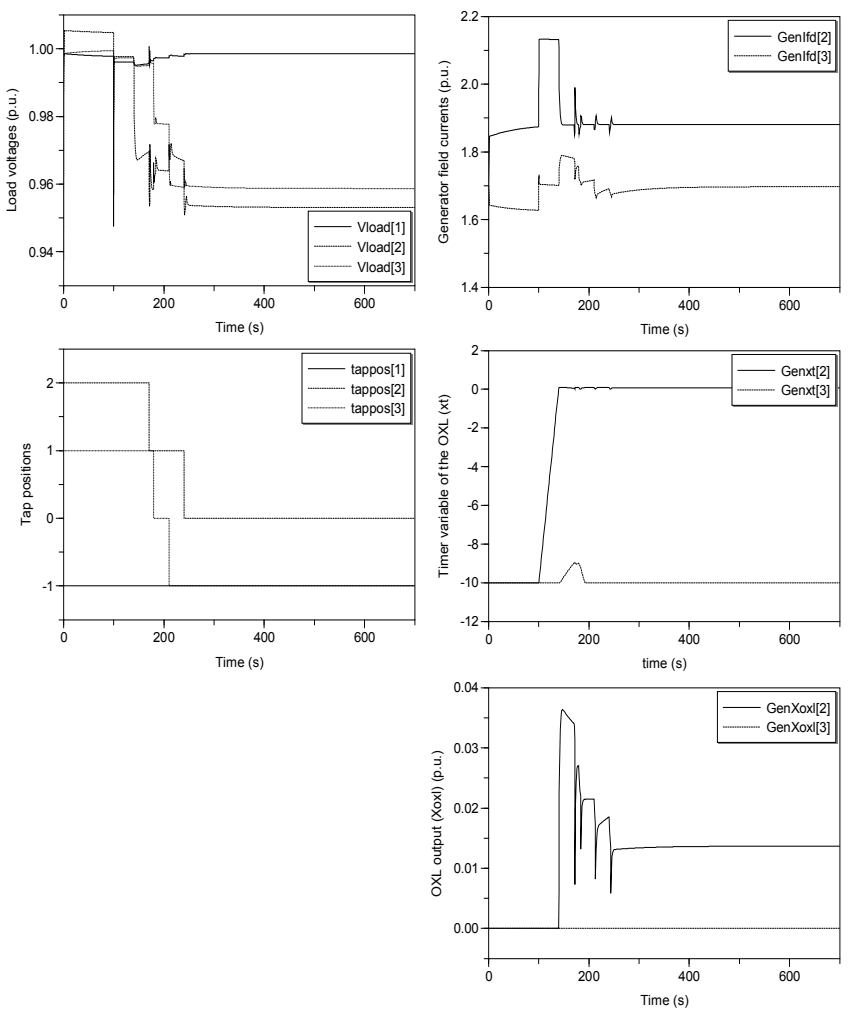

Figure 6. Response to the fault with LTC setpoint reduction

\section{A coordinated application of one step CB switching, LTC setpoint reduction and one step load shedding}

In case the fault considered earlier is followed by another line tripping, one of the lines between areas 2 and 3 at $t=110 \mathrm{~s}$, which is often the case due to a cascade of events in voltage collapse, simulation results, not shown here, show that single strategies such as LTC setpoint reduction, one step CB switching (corresponding to 0.1 p.u. of reactive compensation), one step load shedding (corresponding to disconnection of 10 $\%$ of load) and even a mixture of the LTC setpoint reduction and $\mathrm{CB}$ switching individually fail to arrest the voltage collapse. However, it is possible to stabilize all the load voltages by a coordinated application of (one step) $\mathrm{CB}$ switching, LTC setpoint reduction and (one step) load shedding in areas 1,2 and 3 at the right moment. As shown in figure 7, switching of $\mathrm{CB}$ in area 2 relieves the generator in this area of some reactive power, on the other hand, load shedding in both areas 2 and 3 relieves the generators in these areas of both active and reactive power and as a result keeps the generator field currents well below their limit $\left(\operatorname{Ifd}_{\lim }=1.88\right.$ p.u. for area 2 and $\operatorname{Ifd}_{\lim }=1.75$ p.u. for area 3 ). Notice that both OXLs are inactive in the long-term. 

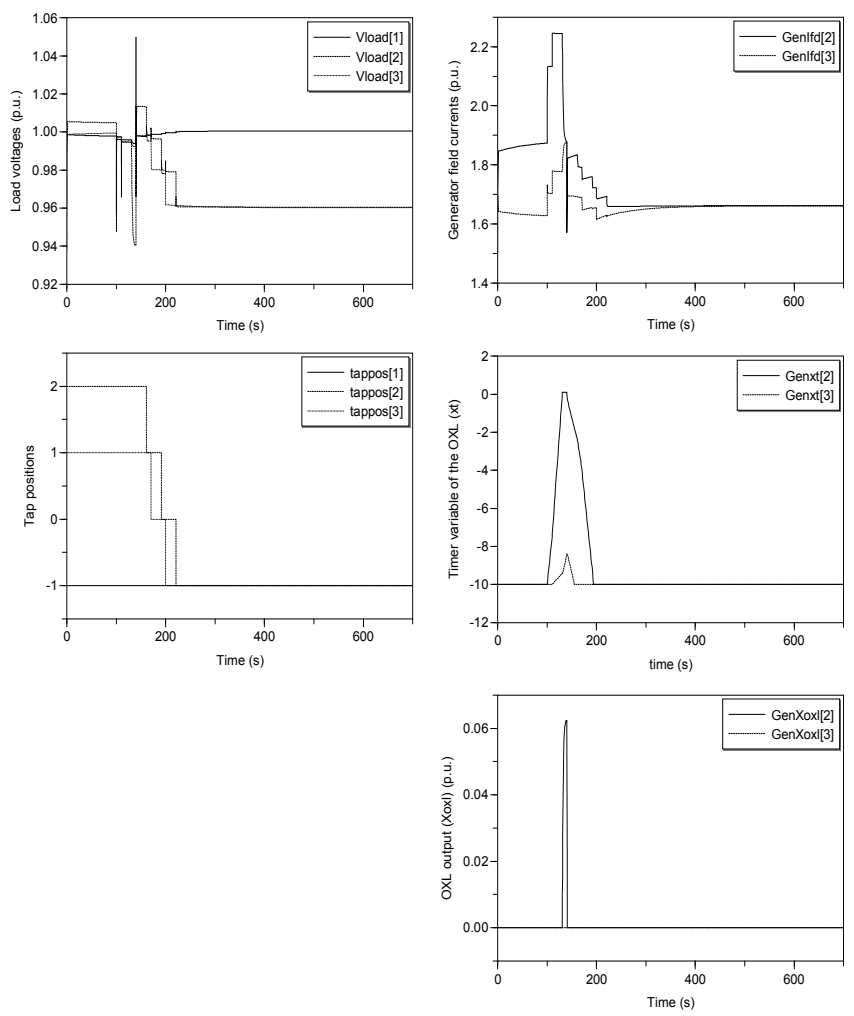

Figure 7. Response to the fault with a coordinated application of $\mathrm{CB}$ switching, LTC setpoint reduction and load shedding

\section{CONCLUSION AND FUTURE WORK}

Logical controllers e.g. LTCs and CBs as well as discrete control logics such as threshold reached by OXLs introduce discrete events into power system continuous dynamics. The resulting dynamic behavior often involves intrinsic interactions between continuous dynamics and discrete events, particularly during voltage collapse phenomena when many discrete devices (either controllers or thresholds) switch on and off. The ordering of these events is very important for the stabilization and is modeled using concurrent execution of hybrid automata.

This paper presented an efficient framework to capture the hybrid behavior of power system using Modelica as an objectoriented equation-based language. For the purpose of coordinated voltage control the Modelica model for transmission lines, LTC, OXL, CB, and dynamic exponential recovery loads has been presented in the hybrid framework. All component models are transparent and can easily be modified or extended.

Simulation results showed that the interaction between local controllers and continuous dynamics of power system as well as nonlinear behavior of load dynamics can easily be studied in the proposed hybrid framework and thus any appropriate coordinated voltage control action to arrest voltage collapse can effectively be analyzed. For the case study considered in this paper, the simulator integration time when running on a $3.15 \mathrm{GHz}$ Intel Core 2 Duo CPU with $4 \mathrm{~GB}$ of RAM takes less than 1 s, i.e. about 700 times faster than real time resulting in a flexible environment for modeling and simulation of large power systems at which any control strategy can easily be tested or verified.

In the current study the timing of the control actions was obtained by carrying out many simulations. In future work this timing of events will be obtained automatically by a Model Predictive Control (MPC) selecting the best scenarios among a small number of possible scenarios. The fact that these scenarios can be simulated over a long time window allows efficient and automatic comparison of their perfor- mance.

\section{ACKNOWLEDGMENT}

This work was supported by the Special Research Fund at Ghent University (BOF) B/06484/01. This paper presents research results of the Belgian Network DYSCO (Dynamical Systems, Control, and Optimization), funded by the Interuniversity Attraction Poles Programme, initiated by the Belgian State, Science Policy Office and EUFP7 project CON4COORD. The scientific responsibility rests with its authors.

\section{REFERENCES}

[1] I.R. Navarro, M. Larsson, G. Olsson, "Object-oriented modeling and simulation of power systems using Modelica", In Proceedings of the IEEE power engineering society, Winter meeting, (pp. 790-795), Singapore, 2000.

[2] Dynasim AB, "Dymola-user's manual", TechnicalReport, Lund, Sweden, 2008.

[3] Modelica Design Group, "Modelica - A unified object-oriented language for physical systems modeling", Tutorial and rationale, http://www.modelica.org/, 1999.

[4] P. Fritzson, "Modelica tutorial- Introduction to object-oriented modeling and simulation with openModelica", 2006.

[5] R.R. Negenborn, S. Leirens and et. al, "Supervisory nonlinear MPC for emergency voltage control using pattern search", Journal of the control engineering practice17 (pp. 841-848), 2009.

[6] A. G. Beccuti and M.Morari, "A hybrid system approach to power systems voltage control," In IEEE CDC and ECC Seville, 2005.

[7] S. Leirens, J. Buisson and et al, " A hybrid approach to the power system voltage stability issue", Technical report, hybrid system control group, France, 2004.

[8] T. Van Cutsem and C.Vournas, "Voltage stability of electric power systems", Power electronics and power systems series, Kluwer academic publishers. ISBN 0-7923-8139-4, 1998.

[9] J. Machowski, J.W. Bialek and J.R. Bumby, "Power system dynamics and stability", ISBN 0-471-97174. Wiley, 1993.

[10] M. Larsson, "ObjectStab - A Modelica library for power system stability studies", Modelica workshop, Lund University, Sweden, 2000.

[11] M. Larsson, "The ABB power transmission test case", Technical report, 2002. 